\title{
EFICACIA DE FLORFENICOL PARA EL TRATAMIENTO DE INFECIONES POR Pasteurela Multocida Y Mannheimia Haemolitica EN LLAMAS
}

\author{
Marcos Ferrante ${ }^{1}$.
}

1. Docente del curso de Medicina Veterinária de la Universidade Estadual de Maringá UEM. marcosferrante@gmail.com

Recebido em: 22/09/2018 - Aprovado em: 23/11/2018 - Publicado em: 03/12/2018 DOI: 10.18677/EnciBio_2018B41

\begin{abstract}
RESUMEN
El florfenicol es uno de los principales antibióticos utilizados en la terapéutica de las infecciones bacterias respiraratorias en la clínica de grandes animales. Sin embargo, no existen estudios farmacocinéticos/farmacodinámicos (PK/PD) que evalúen su eficacia en la antibioticoterapia de llamas. El objetivo del presente trabajo es evaluar el efecto del tratamiento de dosis de $20 \mathrm{mg} / \mathrm{kg}$ por vía intramuscular de florfenicol en el tratamiento de las infecciones causadas por $P$. multocida y $M$. Haemolitica en llamas. Se realizó una simulación de Monte Carlo para determinar las tasas de eficacia. Las tasas de eficacia estimadas para $P$. Multocida fueron del $99 \% ; 20 \%$ y $1 \%$ y según las CIM de las cepas 0,5; 1 y 2 respectivamente. Mientras que las tasas de eficacia estimadas para M. Haemolitica foram del 100\%; 95\% y 3\% y según las CIM de las cepas 0,5; 1 y 2 respectivamente. La probabilidad de obtener la cura bacteriológica después del tratamiento disminuyó significativamente para infecciones causadas por bacterias con CIM superiores a $0,5 \mathrm{ug} / \mathrm{ml}$ para $P$. Multocida $(p 0,01)$ y $1 \mathrm{ug} / \mathrm{ml}$ para $M$. Haemolitica $(p 0,01)$. Estos resultados evidencian la necesidad de incorporar en el protocolo terapéutico el aislamiento bacteriológico, la determinación de la CIM y optimización de la dosi terapéutica según la susceptibilidad da cepas de $P$. multocida yM. Haemoliticaa fin de evitar fallas terapéuticas y consecuentemente potenciar el desarrollo de resistencia bacteriana.
\end{abstract}

PALABRAS CLAVES: Modelamento farmacocientico/farmacodinâmico; Lama glama; camelidos sudamericanos

\section{EFICACY OF FLORFENICOL FOR THE TREATMENT OF INFECTIONS BY Pasteurella Multocida and Mannheimia Haemolitica IN LLAMAS}

\begin{abstract}
Florfenicol is one of the main antibiotics used in the therapy of bacterial respiratory infections in the clinic of large animals. However, there are no pharmacokinetic / pharmacodynamic (PK/PD) studies evaluating its efficacy in llamas antibiotic therapy. The objective of the present study is to evaluate the effect of treatment of intramuscular $20 \mathrm{mg} / \mathrm{kg}$ dose of florfenicol in the treatment of infections caused by $P$. multocida and $M$. Haemolitica in llamas. A Monte Carlo simulation was performed to determine efficacy rates. The estimated efficacy rates for $P$. multocida were $99 \%$; $20 \%$ and $1 \%$ and according to MICs of strains $0.5 ; 1$ and 2 respectively. While the estimated efficacy rates for $\mathrm{M}$. haemolitica were 100\%, 95\% and $3 \%$ and according


to MICs of strains $0.5,1$ and 2 respectively. The probability of obtaining the bacteriological cure after treatment decreased significantly for infections caused by bacteria with MICs greater than $0.5 \mathrm{ug} / \mathrm{ml}$ for $P$. multocida $(p 0,01)$ and $1 \mathrm{ug} / \mathrm{ml}$ for $M$. haemolitica $(p 0,01)$. These results evidenced the need to incorporate in the therapeutic protocol bacteriological isolation, determination of MIC and therapeutic dose optimization according to the susceptibility of strains of $P$. multocida and $M$. Haemolitica in order to avoid therapeutic failures and consequently to enhance the development of Bacterial resistance.

KEYWORDS:Pharmacokinetic / pharmacodynamicmodeling, Lama glama, South American camelids

\section{INTRODUCCIÓN}

La familia de camélidos sudamericanos comprende cuatro especies: llama (Lama glama), alpaca (Lama pacos), guanaco (Lama guanicoe) y vicugna (Vicugna vicugna). La poblacion total estimada para Argentina, Chile, Bolívia y Peru es de 3,2 millones de alpacas y 3,6 millones de llamas (CARDOZO, 1981). La cantidad de pequeños productores de en estos países llega Más de un millón de pequeños productores de los Andes centrales de Sudamérica tienen alpacas (Vicugna pacos) y llamas (Lama glama) como principal medio de subsistencia. Los animales proveen principalmente carne, leche y fibra, y además, son considerados parte fundamental de la identidad cultural de sus pueblos (QUISPE, et al.; 2009, LEDESMA et al. 2017).

Por lo tanto, su estado sanitario debe estar bien establecido. Según el informe publicado por la la OIE em 2008 sobre "Enfermedades de los camélidos (Organización Mundial de Sanidad Animal), las infecciones respiratorias producidas principalmente por pasteurellaspp. han sido reportados para los camélidos. La pasteurelosis se clasificó dentro del grupo III por ser considerada uma enfermedad leve (OIE, 2008).

Segun un estúdio realizado por Fassi-Fehri (1987) en diversos países del

mundo Pasteurela. Multocida ( $P$. Multocida) es la bactéria oportunista mas frequentemente asociada en las enferemedades respiratórias en las espécies de la familia Camelidade. Por outro lado, estúdios mas recientes reportaron altos niveles de seroconversion en camelidos sudamericanos para P.multocida y para Mannheimia haemolitica (M. haemolitica) en 6 provincias de Argentina. Estos resultados indican que estas bacterias poderian estar causando infecciones subclinicas en estas espécies (DÍAZ et al., 2017; LEDESMA et al., 2017). Mientras que en Peru fueron reportados casos de muertes de alpacas por neumonia aguda. Em estos casos, fue confirmado la presencia los virus Parainfluenza 3 (PI3), respiratorio sincitial bovino (RSB) y adenovirus bovino tipo 3 (BAdV-3). Coexistindo con estos vírus fueron determinadas las bactérias $P$. multocida y M.haemolytica (ROSADIO et al., 2011; CIRILO et al., 2012; GUZMAN, et al., 2013). Posteriormente, Rímac et al.; (2017) revelaron la detección de P.multocida tipo A, LPS genotipo L6 y tox $A+y \operatorname{tbp} A+$ de alpacas jóvenes muertas con neumonía en Perú.

Una de las principales alternativas terapéuticas de las infecciones bacterianas respiratorias en bovinos y suinos es el antibiótico florfenicol (SIDHU et al., 2014; DOREY et al., 2017). Este antimicrobiano sintético de amplio espectro desarrollado especialmente para uso veterinario se deriva del tianfenicol, que contiene un átomo de flúor en el carbono tres en lugar del grupo hidroxilo (DOREY et al., 2017).

Los modelos PK/PD son ampliamente utilizados como indicadores de eficacia de los tratamientos antibióticos en humanos y animales (EMA, 2016; LINDER et al., ENCICLOPÉDIA BIOSFERA, Centro Científico Conhecer - Goiânia, v.15 n.28; p. 472 2018 
2016). La utilización de estos modelos conjuntamente con el método de simulación de Monte Carlo fue descrita por Dudley et al., (2000) y Trang et al., (2017). Actualmente esta es la principal metodología empleada para la evaluación y optimización de dosis terapéuticas de los antibióticos (REY et al., 2014; EMA, 2016; TOUTAIN et al., 2016b; TOUTAIN et al., 2016a; WEl et al., 2017).

En el caso del antibiótico florfenicol se ha demostrado que los índices PKPD adecuados son el área bajo la curva de concentración plasmática hasta 24 horas después de la administración sobre la concentración inhibitoria mínima $\left(\mathrm{AUC}_{24} / \mathrm{CIM}\right)$ (AHMAD et al., 2016). Este índice permite determinar si una dosis administrada resultara en efecto bacteriotatico y el efecto bactericida o erradicación bacteriológica, considerando esta última cuando la reducción en el número de bacterias es mayor de 4 log (DOREY et al., 2017). De esta forma los modelos PKPD permiten identificar fallas en las dosis terapéuticas que incrementan el riesgo de selección de cepas resistentes.

Existen estudo de farmacocientica realizados por Pentecost et al., (2013) adminsitrando la dosis de $20 \mathrm{mg} / \mathrm{kg}$ de florfenicol en llamas, sin embargo, los paramentos PK no fueron utilizados para evaluar la eficacia de esta dosis en el tratamiento de las infecciones causadas por P. multocidayM. Haemolitica. El objetivo del presente trabajo es evaluar el efecto del tratamiento en las dosis de $20 \mathrm{mg} / \mathrm{kg}$ por vía intramuscular de florfenicol en el tratamiento de las infecciones causadas por $P$. multocida y M. Haemolitica en llamas utilizando el modelado PK/PD.

\section{MATERIALES Y MÉTODOS}

Se evaluó la eficacia del tratamiento por vía intramuscular de florfenicol en el tratamiento de infecciones bacterianas causadas por P. multocida y M. Haemolitica. Se realizó una simulación de Monte Carlo de los parámetros PK de 10.000 eventos, a continuación se realizó el modelado PK/PD para determinar las tasas de eficacia en la metodología descrita por Dudley et al., (2000) y Trang et al., (2017). Los parámetros farmacocinéticos utilizados fueron obtenidos del estudio realizado por Pentecost et al., (2013). La dosis fue de $20 \mathrm{mg} / \mathrm{kg}$ de florfenicol vía intramuscular y la $A B C_{24}$ de la concentración plasmática fue de $37,8 \pm 6.8 \mathrm{ug} / \mathrm{mL}$ (FIGURA 1). El parámetro de eficacia utilizado fue el índice del área sube la curva de la concentración plasmática en 24 horas/concentración inhibitoria mínima $\left(\mathrm{ABC}_{24} / \mathrm{CIM}\right)$ (TOUTAIN et al., 2007; AHMAD et al., 2016) (FIGURA 2). Los índices $A B C_{24} / \mathrm{CIM}$ para efecto bacteriostático fueron de 10 y 14; para efecto bactericida fueron de 24 y 43 y para erradicación fue de 36 y 63 para $P$. multocida y $M$. Haemolitica respectivamente. Os cálculos fueron realizados para cepas com CIM en el intervalo de $0.125 \mathrm{ug} / \mathrm{ml}$ hasta $8 \mathrm{ug} / \mathrm{ml}$ (SIDHU et al., 2014). El análisis de los datos y la simulación de Monte Carlo se realizó en el programa Microsoft Excel (ODA, 2011; SHARMA et al., 2016) y en el programa BioEstat 5.3. Para la comparación de las tasas de eficacia se utilizó la prueba de chi-cuadrado con una significancia de ( $p$ $0,05)$.

\section{RESULTADOS}

Se observaron cambios en las tasas de eficacia relacionadas con los cambios en la CIM (FIGURA 3 y 4). Las tasas de eficacia estimadas para $P$. Multocida fueron de $99 \% ; 20 \%$ y $1 \%$ y según las CIM de las cepas 0,$5 ; 1$ y 2 respectivamente. La probabilidad de alcanzar el índice de erradicación bacteriológica después del tratamiento disminuyó significativamente para infecciones causadas por bacterias con CIM superiores a $0,5 \mathrm{ug} / \mathrm{ml}(p \quad 0,01)$. Mientras que para $M$. Haemolitica fueron del $100 \% ; 95 \%$ y $3 \%$ y según las CIM de las cepas 0,$5 ; 1$ y 2 respectivamente. La ENCICLOPÉDIA BIOSFERA, Centro Científico Conhecer - Goiânia, v. 15 n.28; p. 473 2018 
probabilidad de alcanzar el índice de erradicación bacteriológica después del tratamiento disminuyó significativamente para infecciones causadas por bacterias con CIM superiores a $1 \mathrm{ug} / \mathrm{ml}(p \quad 0,01)$.

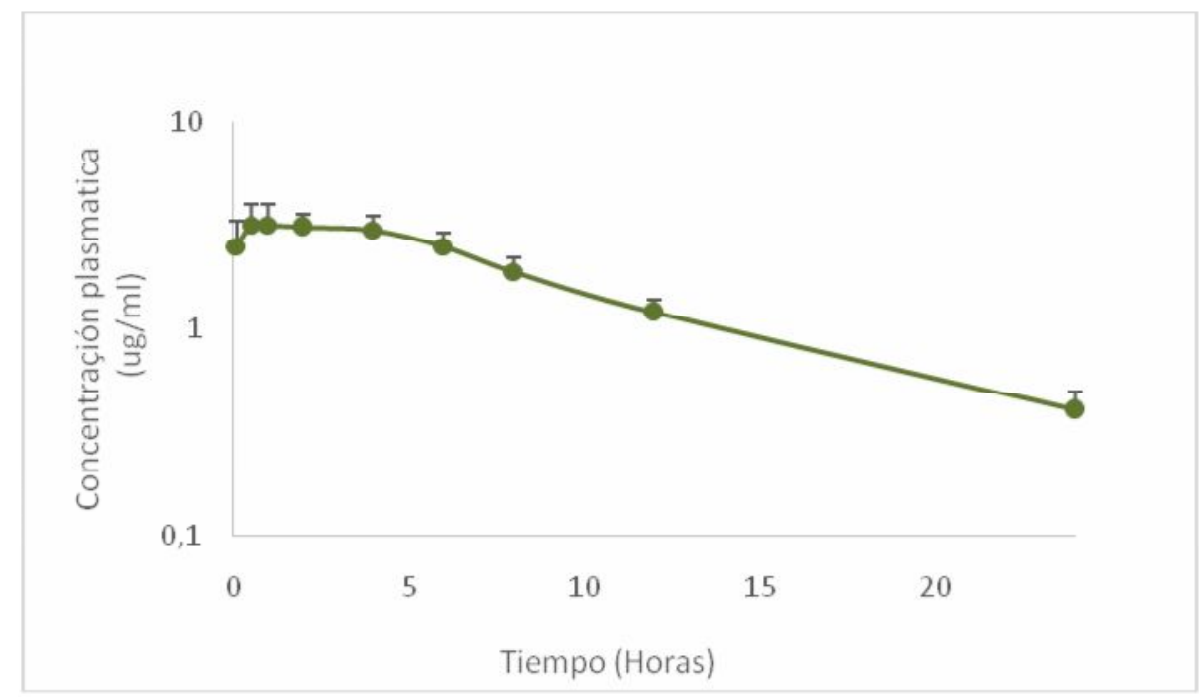

FIGURA 1.Farmacocinética plasmática de la administración de $20 \mathrm{mg} / \mathrm{kg}$ vía intramuscular de florfenicol en llama.

FONTE: Penecost, et al., (2013).

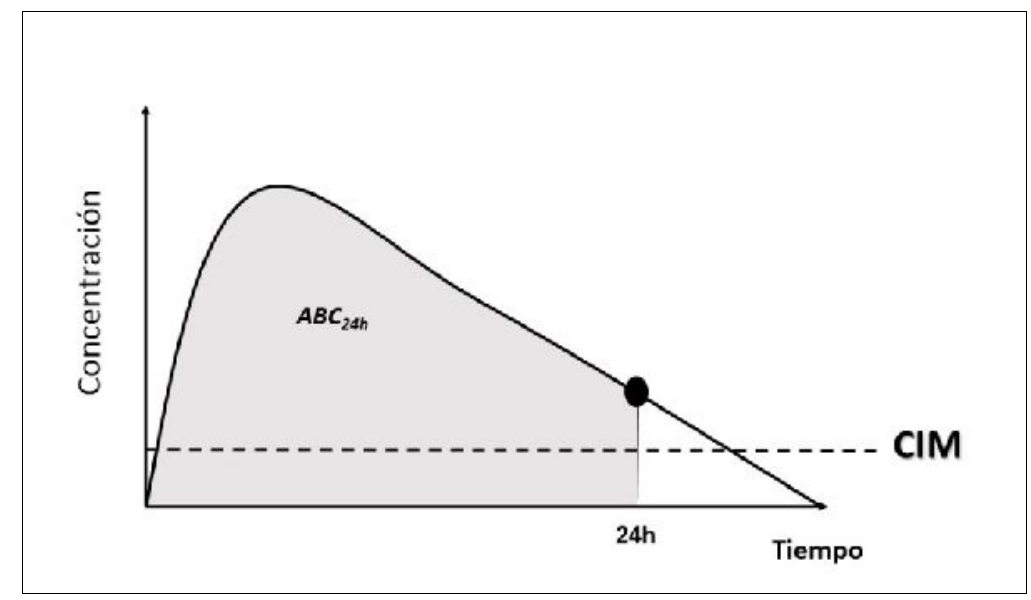

FIGURA 2. Relación de los parametros PK y PD. El parametro de PK corresponde a la área bajo la curva de tiempo de concentración plasmática de 0 a $24 \mathrm{~h}\left(\mathrm{AUC}_{24}\right)$ y $\mathrm{El}$ parâmetro de PD corresponde a la concentración mínima inhibitoria (CIM).

FONTE: AHMAD et al., 2016. 


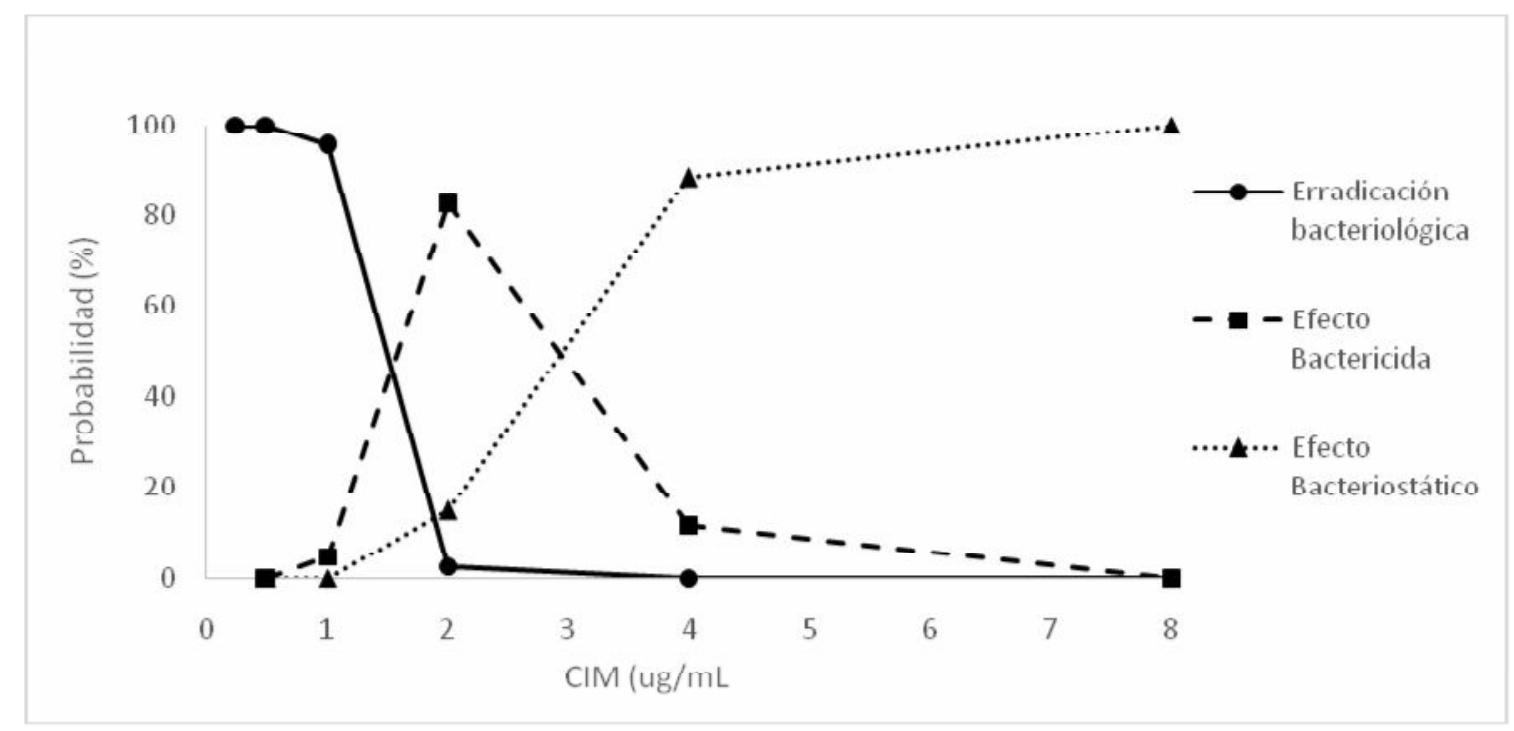

FIGURA 3: Probabilidad de alcanzar el índice $\mathrm{ABC}_{24} / \mathrm{CIM}$ segun la $\mathrm{CIM}$ de la cepa de $P$. multocida infectante luego del tratamiento con $20 \mathrm{mg} / \mathrm{kg}$ via intramuscular de florfenicol en llamas. Baseado en la simulação de Monte Carlo $(n=10.000)$.

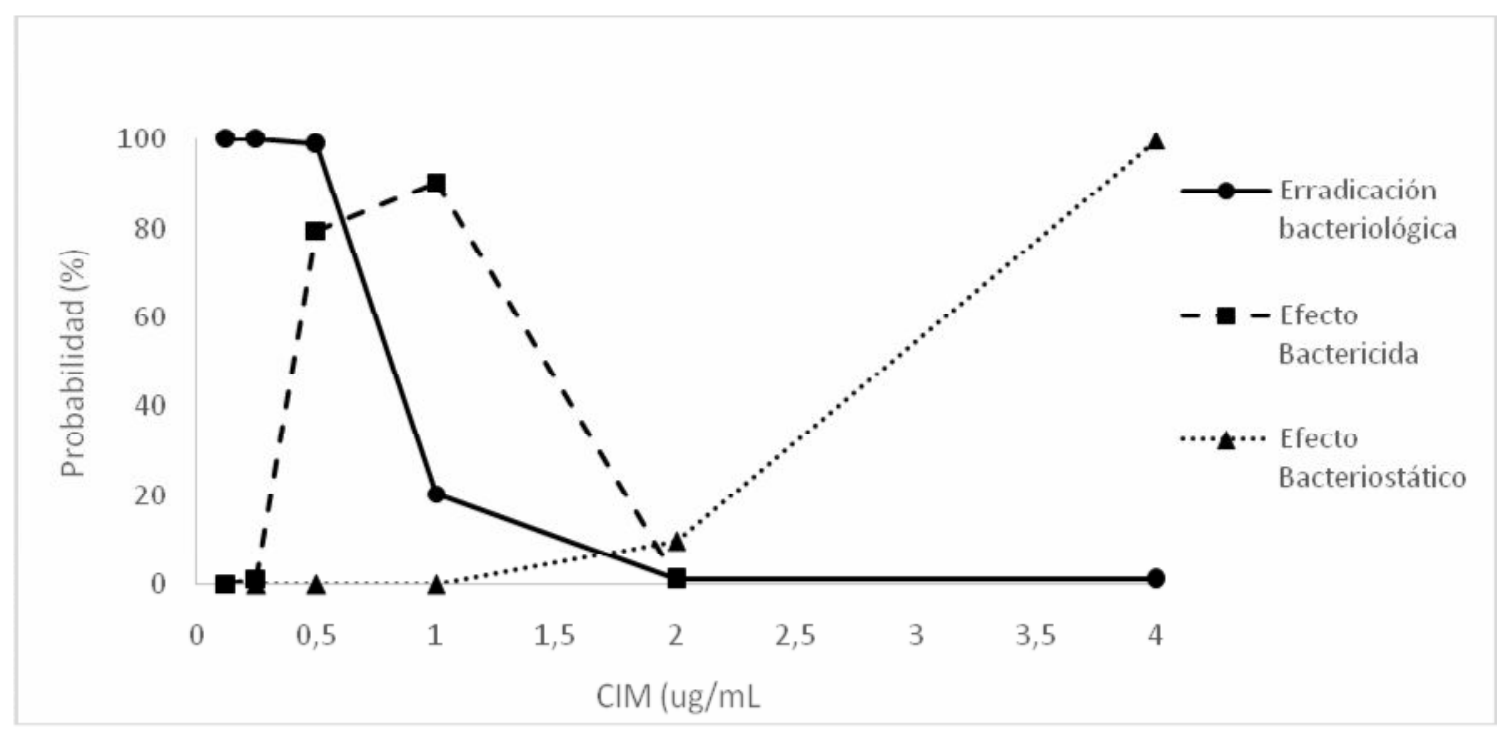

FIGURA 4: Probabilidad de alcanzar el índice $\mathrm{ABC}_{24} / \mathrm{CIM}$ segun la $\mathrm{CIM}$ de la cepa de $M$. Haemolitica infectante luego del tratamiento con $20 \mathrm{mg} / \mathrm{kg}$ via intramuscular de florfenicol en llamas. Baseado na simulação de Monte Carlo $(n=10.000)$.

\section{DISCUSIÓN}

El modelo PK/PD permitió estimar la eficacia del tratamiento con $20 \mathrm{mg} / \mathrm{kg}$ de florfenicol vía intramuscular según la bacteria causante de la infección considerando los valores de CIM. El éxito terapéutico de la antibioticoterapia depende principalmente de la relación entre la concentración del fármaco en el sitio de infección con la concentración inhibitoria mínima de la bacteria causante de la infección (TOUTAIN et al., 2016a; AHMAD et al., 2016). Por lo tanto, la falla terapéutica podría ser de origen PK, PD o por una combinación de ambos. Las fallas terapéuticas de origen PK se presentan cuando el fármaco no alcanza la concentración necesaria en el sitio de acción y las de origen PD cuando la bacteria resiste las concentraciones terapéuticas originales (TORNEKE y TORREN-DO, 2015; HU y CHENG 2016; TOUTAIN et al., 2016b). Por lo tanto, la elección de la 
doses terapéutica debe ser baseada considerando la CIM de los microorganismos causantes de la infección para el fármaco seleccionado (BOOTHE y BOOTHE, 2015). En este sentido los análisis de eficacia según la CIM establece la información complementaria para el uso racional de antibióticos (REY et al., 2014; WEl et al., 2017). El presente estudio, permitió determinar las bajas tasas de erradicación bacteriológica de esta dosis para el tratamiento de las infecciones causadas por $P$. multocida y $M$. Haemolitica con CIM superiores a 0,5 y $1 \mathrm{ug} / \mathrm{ml}$ respectivamente (FIGURA 5). Por lo tanto, son necesarios estudios de ajustes a dosis terapéuticas que permitan obtener tasas de erradicación bacteriológica adecuadas.

\section{CONCLUSIÓN}

El modelado PK/PD permitió estimar el efecto subterapéutico de las dosis de $20 \mathrm{mg} / \mathrm{kgn}$ de florfenicol vía intramuscular en llamas, demostrando la necesidad de optimización de las dosis terapéuticas. Complementariamente, este estudio demuestra que pequeños incrementos en la CIM determinan grandes cambios en las tasas de eficacia. Esto evidencia la necesidad de incorporar en el protocolo terapéutico el aislamiento bacteriológico, la determinación de la CIM y optimización de dosis terapéutica según la susceptibilidad bacteriana a fin de evitar fallas terapéuticas y consecuentemente potenciar el desarrollo de resistencia bacteriana.

\section{REFERENCIAS}

AHMAD, I.; HUANG, L.; HAO, H.; SANDERS, P.; YUAN, Z. Application of PK/PD Modeling in Veterinary Field: DoseOptimization and DrugResistancePrediction. BioMed Research International, vol. 2016, 12 p. 2016. Disponible en: <http://dx.doi.org/10.1155/2016/5465678> Acesso em: 17 mar. 2018.

BOOTHE, D. M.; BOOTHE, H. W. Antimicrobial Considerations in the Perioperative Patient. Veterinary Clinics: Small Animal Practice, v. 45, n. 3, p. 585-608, 2015. Disponible en: https://doi.org/10.1016/j.cvsm.2015.01.006 . Acesso en: 15 may. 2018.

CARDOZO, A. Espécies zootécnicas nativas de los Andes Altos. In: Organizacion de las Naciones Unidas para la Agricultura y la Alimentacion - FAO (Org.). Recursos genéticos animales en América Latina: ganado criollo y especies de altura. Roma: FAO y PNUMA, 1981. cap. IX. Disponible en: http://www.fao.org/docrep/009/ah223s/AH223S10.htm . Acesso en: 25 jun. 2018

CIRILO, E.; MANCHEGO, A.; RIVERA, H.; ROSADIO, R. Coexistencia de virus y bacterias en neumonías agudas en alpacas neonatas. Revista de Investigaciones Veterinarias del Perú, v. 23, n. 3, p. 317-335, ago. 2012. Disponible en: http://dx.doi.org/10.15381/rivep.v23i3.914 . Acesso en: 19 jul. 2018

DÍAZ, A. M.; LEDESMA, M. M.; CALCAGNO, M. L.; LEONI, J.; MANGHI, M. A. et al. Serological Survey of Antibodies to Mannheimia haemolytica and Pasteurellamultocida in Camelids from Argentina. Annals of Infectious Disease and Epidemiology, v. 2, n. 4, p. 1 - 6, 2017. Disponible en: http://www.remedypublications.com/infectious-disease-andepidemiology/articles/pdfs_folder/aide-v2-id1024.pdf . Acesso en: 20 feb. 2018 
DOREY, L.; PELLIGAND, L.; CHENG, Z.; LEES, $P$. Pharmacokinetic/pharmacodynamics integration and modelling of florfenicol for the pig pneumonia pathogens Actinobacillus pleuropneumoniae and Pasteurella multocida. PLoS ONE, v. 12, n. 5, 2017. Disponible en: https://doi.org/10.1371/journal.pone.0177568 . Acesso en: 20 feb. 2018.

DUDLEY, M. N.; AMBROSE, P. G. Pharmacodynamics in the study of drug resistance and establishing in vitro susceptibility breakpoints: ready for prime time. Current Opinion in Microbiology, v. 3, n. 5, p. 515-521, 2000. Disponible en: https://doi.org/10.1016/S1369-5274(00)00132-6 . Acesso en: 15 jan. 2018

EUROPEAN MEDICINE AGENCY-EMA. (London) Guideline on the Use of Pharmacokinetics and Pharmacodynamics in the Development of Antimicrobial Medicinal Products. 2016.2 Disponible en: http://www.ema.europa.eu/docs/en_GB/document_library/Scientific_guideline/2016/ 07/WC500210982.pdf . Acesso en: 18 jun. 2018.

FASSI-FEHRI, M. M. Les maladies des camelides [Diseases of camelids]. Revue scientifique et technique (International Office of Epizootics), v. 6, n. 2, p. 315335, 1987. Disponible en: https://www.doc-developpementdurable.org/file/Elevages/chameau-

dromadaire/Les\%20maladies\%20des\%20cam\%C3\%A9lid\%C3\%A9s.pdf . Acesso em: 30 abr. 2018.

GUZMÁN, M. K.; ROSADIO, R.; LENIN, M. H.; MANCHEGO, A. Asociation of bacterial and viral agents in acute pneumonia in young alpacas. Revista de Investigaciones Veterinarias del Perú, v. 24, n. 4, p. 524-536, 2013. Disponible en: http://www.scielo.org.pe/scielo.php?script=sci_abstract\&pid=S160991172013000400015\&lng=es\&nrm=iso . . Acesso em: 19 jun. 2018.

HU, Y.; CHENG, H. Health Risk from Veterinary Antimicrobial Use in China's Food Animal Production and Its Reduction. Environmental Pollution, v. 219, p. 993-997, 2016. Disponible en: https://doi.org/10.1016/j.envpol.2016.04.099. Acesso em: 28 jun. 2018.

LEDESMA, M. M.; DÍAZ, A. M.; BARBERIS, C.; VAY, C.; MANGHI, M. A. et al. Identification of Lama glama as Reservoirs for Acinetobacterlwoffii. Frontiers in microbiology, v. $8, \quad$ p. 278, 2017. Disponible en: https://www.ncbi.nlm.nih.gov/pmc/articles/PMC5332404/pdf/fmicb-08-00278.pdf . Acesso en: 11 may. 2018. DOI: 10.3389/fmicb.2017.00278

LINDER, K. E.; NICOLAU, D. P.; NAILOR, M. D. Predicting and Preventing Antimicrobial Resistance Utilizing Pharmacodynamics: Part I Gram Positive Bacteria. Expert opinion on drug metabolism \& toxicology, v. 12, n. 3, p. 267-280, 2016. Disponível em: < https://doi.org/10.1517/17425255.2016.1141197>. Acesso em: 08 ago. 2018.

ODA, K. Development of Software for Antimicrobial PK/PD Simulation incorporating Montecarlo Simulation Based on Microsoft® Office Excel. Journal of Pharmaceutical Health Care and Sciences, v. 37, p. 335-344, 2011. Disponible en: https://doi.org/10.5649/jjphcs.37.335 . Acesso en: 12 jul. 2018. 
OIE. (Paris). Report of the ad hoc group on diseases of camelids. 2008. Disponible en: http://www.oie.int/downld/SC/2008/A_BSC_sept2008.pdf . Acesso en: 16 jun. 2018.

PENTECOST, R. L.; NIEHAUS, A. J.; WERLE, N. A.; LAKRITZ, J. Pharmacokinetics of florfenicol after intravenous and intramuscular dosing in llamas. Research in veterinary science, v. 95, n. 2, p. 594-599, 2013. Disponible en: <https://doi.org/10.1016/j.rvsc.2013.05.009>. Acesso en: 06 jan. 2018.

QUISPE, E. C.; RODRIGUEZ, T. C.; IÑIGUEZ, L. R.; MUELLER, J. P. Producción de fibra de alpaca, llama, vicuña y guanaco en Sudamérica. Animal Genetic Resources/Resources génétiques animales/Recursos genéticos animales, $v$. 45 , p. 1-14, 2009. Disponible en: http://infoalpacas.com.pe/wpcontent/uploads/2014/07/Fibra-de-alpaca.pdf . Acesso en: 06 jan. 2018. Doi: $10.1017 / \mathrm{S} 1014233909990277$

REY, J. F.; LAFFONT, C. M.; CROUBELS, S.; BACKER, P.; ZEMIRLINE, C. et al. Use of Monte Carlo simulation to determine pharmacodynamiccutoffs of amoxicillin to establish a breakpoint for antimicrobial susceptibility testing in pigs. American journal of veterinary research, v. 75, n. 2, p. 124-131, 2014. Disponible en: https://doi.org/10.2460/ajvr.75.2.124 . Acesso en: 24 mar. 2018

RÍMAC, R.; LUNA, L.; HURTADO, R.; ROSADIO, R.; MATURRANO, L. Detection and genetic characterization of Pasteurellamultocida from alpaca (Vicugnapacos) pneumonia cases. Tropical animal health and production, v. 49, n. 6, p. 13251328, 2017. Disponible en: 10.1007/s11250-017-1309-5 . DOI: 10.1007/s11250017-1309-5. Acesso en: 05 abr. 2018.

ROSADIO, R.; CIRILO, E.; MANCHEGO, A.; RIVERA, H. Respiratory syncytial and parainfluenza type 3 viruses coexisting with Pasteurella multocida and Mannheimia hemolytica in acute pneumonias of neonatal alpacas. Small ruminant research, v. 97, n. 1-3, p. 110-116, 2011. Disponible en: https://doi.org/10.1016/j.smallrumres.2011.02.001 . Acesso en: 05 abr. 2018.

SHARMA, V. D.; SINGLA, M.; CHAUDHARY, M.; KUMAR, M.; BHATNAGAR, A. et al. Dose optimization of ceftriaxone-vancomycin combination using fractional inhibitory concentration kinetics in resistant bacteria. ADMET and DMPK, v. 4, n. 3, p. 241-260, 2016. Disponible en: https://hrcak.srce.hr/167045 . Acesso en: 25 may. 2018. Doi: $10.5599 /$ admet.4.3.286

SIDHU, P.; RASSOULI, A.; ILLAMBAS, J.; POTTER, T.; PELLIGAND, L. et al. Pharmacokinetic-pharmacodynamic integration and modelling of florfenicol in calves. Journal of veterinary pharmacology and therapeutics, v. 37, n. 3, p. 231-242, 2014. Disponible en: https://onlinelibrary.wiley.com/doi/abs/10.1111/jvp.12093 . Acesso en: 25 may. 2018. DOI: 10.1111/jvp.12093.

TÖRNEKE, K.; TORREN-EDO, J.; GRAVE, K.; MACKAY, D. K. J The management of risk arising from the use of antimicrobial agents in veterinary medicine in EU/EEA countries-a review. The management of risk arising from the use of antimicrobial agents in veterinary medicine in EU/EEA countries-a review. Journal of veterinary pharmacology and therapeutics, v. 38, n. 6, p. 519-528, 2015. Disponible en: $<$ https://doi.org/10.1111/jvp.12226>. Acesso en: 21 jul. 2018. 
TOUTAIN, P. L.; BOUSQUET-ME, A; MARTINEZ, M. AUC / MIC : A PK / PD Index for Antibiotics with a Time Dimension or Simply a Dimensionless Scoring Factor? Journal of Antimicrobial Chemotherapy, v. 60, n. 6, p. 1185-1188, 2007. Disponible en: <https://doi.org/10.1093/jac/dkm360>. Acesso en: 21 jul. 2018.

TOUTAIN, P. L.; POTTER, T.; PELLIGAND, L.; LACROIX, M.; ILLAMBAS, J. et al. Standard PK/PD Concepts Can Be Applied to Determine a Dosage Regimen for a Macrolide: The Case of Tulathromycin in the Calf. Journal of Veterinary Pharmacology and Therapeutics, v. 40, n. 1, p. 16-27, 2016a. Disponible en: https://doi.org/10.1111/jvp.12333 . Acesso en: 19 jun. 2018.

TOUTAIN, P. L.; FERRAN, A. A.; BOUSQUET-MELOU, A.; PELLIGAND, L.; LEES, $P$. Veterinary Medicine Needs New Green Antimicrobial Drugs. Frontiers in microbiology, v. 7, p. 1196, 2016b. Disponible en: https://doi.org/10.3389/fmicb.2016.01196 . Acesso en: 16 jun. 2018.

TRANG, M.; DUDLEY, M.; BHAVNANI, S. M. Use of Monte Carlo simulation and considerations for PK-PD targets to support antibacterial dose selection Current opinion in pharmacology, v. 36, p. 107-113, 2017. Disponible en: https://doi.org/10.1016/j.coph.2017.09.009 . Acesso en: 08 jul. 2018.

WEI, Y.; JINRU, J.; TINGTING, X.; CH et al. Drug Design, Determining optimal dosing regimen of oral administration of dicloxacillin using Monte Carlo simulation. Drug design, development and therapy, v. 11, p. 1951, 2017. Disponible en: http://dx.doi.org/10.2147/DDDT.S139632 . Acesso en: 08 jul. 2018. 